\title{
Turbulent radial mixing in the solar nebula as the source of crystalline silicates in comets
}

\author{
D. Bockelée-Morvan ${ }^{1}$, D. Gautier ${ }^{1}$, F. Hersant ${ }^{1}$, J.-M. Huré ${ }^{1,2}$, and F. Robert ${ }^{3}$ \\ 1 Observatoire de Paris, 5 place Jules Janssen, 92195 Meudon, France \\ 2 Université de Paris 7, 2 place Jussieu, 75251, Paris Cedex 05, France \\ 3 Laboratoire de Minéralogie, Muséum d'Histoire Naturelle, 61 rue Buffon, 75005 Paris, France
}

Received 3 October 2001 / Accepted 9 January 2002

\begin{abstract}
There is much debate about the origin of crystalline silicates in comets. Silicates in the protosolar cloud were likely amorphous, however the temperature of the outer solar nebula was too cold to allow their formation in this region by thermal annealing or direct condensation. This paper investigates the formation of crystalline silicates in the inner hot regions of the solar nebula, and their diffusive transport out to the comet formation zone, using a turbulent evolutionary model of the solar nebula. The model uses time-dependent temperature and surface density profiles generated from the 2-D $\alpha$-disk model of Hersant et al. (2001). It is shown that turbulent diffusion is an efficient process to carry crystalline silicates from inner to outer disk regions within timescales of a few $10^{4} \mathrm{yr}$. The warmest solar nebula models which reproduce the $\mathrm{D} / \mathrm{H}$ ratios measured in meteorites, comets, Uranus and Neptune (Hersant et al. 2001) provide a mass fraction of crystalline silicates in the Jupiter-Neptune region in agreement with that measured in comet Hale-Bopp.
\end{abstract}

Key words. solar system: formation - comets: general - comets: individual: C/1995 O1 (Hale-Bopp) planetary systems: fonction - planetary systems: protoplanetary disks

\section{Introduction}

The origin of cometary silicates is controversial. Infrared spectra obtained from ground-based telescopes and the Infrared Space Observatory (ISO) have shown that Mgrich olivines and pyroxenes are present in cometary grains in both amorphous and crystalline form (Hanner et al. 1994; Hayward et al. 2000; Crovisier et al. 1997, 2000; Wooden et al. 1999). Since the formation of crystalline silicates requires high temperatures, in contrast to the amorphous variety, the puzzling question is how comets could have incorporated both high- and low temperature materials, including ices.

Crystalline silicates are encountered in various environments. They are found in interplanetary dust particles (IDPs), sometimes coexisting with the amorphous form in the aggregate IDPs thought to be of cometary origin (Bradley et al. 1992, 1999). Silicates in chondritic meteorites, formed in the inner hot solar nebula, are entirely crystalline. Crystalline silicates have been detected in disks around pre-main-sequence Herbig Ae/Be stars (e.g., Malfait et al. 1998; Bouwman et al. 2001). These disks, surrounding intermediate-mass stars $\left(2-20 M_{\odot}\right)$,

Send offprint requests to: D. Bockelée-Morvan, e-mail: dominique.bockelee@obspm.fr are believed to be similar to the primitive solar nebula from which the Solar System was formed. Crystalline silicates are also found in the debris disks present around young main sequence stars as, e.g., $\beta$-Pic (Knacke et al. 1993). On the other hand, there is no spectral evidence for their presence in the diffuse interstellar medium or molecular clouds, nor in young stellar objects (Demyk et al. 1999; Hanner et al. 1998). It is thus likely that the crystalline silicates present in cometary grains were produced during or after the collapse of the presolar cloud. Crystalline silicates can form at high temperature by direct condensation or thermal annealing of amorphous silicates. This latter process requires temperatures of at least $800 \mathrm{~K}$ (e.g., Gail 1998). The detection of silica $\mathrm{SiO}_{2}$ in Herbig $\mathrm{Ae} / \mathrm{Be}$ systems and comets is consistent with compositional changes associated to thermal annealing of amorphous grains (Bouwman et al. 2001).

We follow the current opinion that silicates infalling from the presolar cloud onto the nebula discoid were all amorphous. Chick \& Cassen (1997) studied the thermal processing of silicates during the collapse of the presolar cloud in order to assess their survivability. Combining results of envelope radiative heating with heating in the accretion shock above the disk (Neufeld \& Hollenback 1994), they show that grains of silicates infalling at distances 
larger than 1 AU from the Sun escaped vaporization. From the temperature radial profiles they compute, we can deduce that, during this stage, the formation of crystalline silicates by annealing of amorphous silicates was inefficient at distances greater than 2-3 AU.

Gail (1998) improved the analysis of the problem. Assuming that the nebula was already formed, he studied the fate of amorphous silicates radially infalling onto the Sun. Using a stationary model of the nebula, he showed that the annealing of amorphous silicates was inefficient at heliocentric distances greater than 1.5 AU. In other words, the temperature was too cold in the outer nebula where comets are currently supposed to have been formed, for the interstellar silicate grains to be thermally processed prior to their incorporation to comets. Therefore, either crystallization was initiated by a low temperature process, as proposed for disks around evolved stars (Molster et al. 1999), or there was a significant radial mixing between the warm and cold parts of the solar nebula. However, Gail (1998) did not take into account in his calculations any mixing process. More recently, Gail (2001) studied mixing by turbulent diffusion in a stationary Keplerian $\alpha$-disk.

We investigate here the thermal annealing of amorphous silicates in the hot regions of the solar nebula and their diffusive transport out to the comet formation zone by using a turbulent evolutionary model of the nebula. In fact, large-scale radial mixing in the nebula has previously been shown to be a requisite in order to fit measurements of the $\mathrm{D} / \mathrm{H}$ ratio in water in LL3 meteorites and Oort cloud comets (Drouart et al. 1999; Mousis et al. 2000). While these authors used a 1-D turbulent model, we take advantage here of the recent 2-D evolutionary model of Hersant et al. (2001) which provides an improved description of the nebula. The model generates time-dependent temperature, density, and pressures profiles throughout the nebula. Hersant et al. further studied the radial distribution and time evolution of the deuterium enrichment in water in the solar nebula due to isotopic exchange with $\mathrm{H}_{2}$ (the main reservoir of deuterium) and diffusive turbulent transport. Using the $\mathrm{D} / \mathrm{H}$ values measured in LL3 meteorites and Oort cloud comets, they constrained the parameters of the nebula, namely its initial accretion rate $\dot{M}_{0}$, initial outer radius $R_{\mathrm{D} 0}$, and the coefficient of turbulent viscosity $\alpha$ (Shakura \& Sunyaev 1973).

The goal of the present paper is to investigate whether the solar nebula models selected by Hersant et al. (2001), which fit the $\mathrm{D} / \mathrm{H}$ ratio in comets, are also able to explain the amount of crystalline silicates detected in these objects. Section 2 summarizes observational constraints obtained on the nature of silicates in comets. The model is presented in Sect. 3 and the results are discussed in Sect. 4.

\section{Cometary silicates}

Measurements concerning cometary silicates are reviewed in detail by Hanner et al. (1997) and Hanner (1999). A summary is given here.
The nature of cometary silicates was mainly investigated from their Si-O stretching bands falling around $10 \mu \mathrm{m}$. Low resolution $8-13 \mu \mathrm{m}$ spectra have been acquired for a dozen comets (see Hanner et al. 1994; Hanner 1999 and references therein). Strong structured emission features were observed for half of them. The detection of a peak at $11.2-11.3 \mu \mathrm{m}$ in $1 \mathrm{P} /$ Halley and some of the later observed comets pointed out the presence of Mg-rich crystalline olivines mixed with Fe-bearing amorphous olivines and pyroxenes (Hanner et al. 1994; Wooden et al. 1999).

Observations of comet C/1995 O1 (Hale-Bopp) provided new insights on silicate mineralogy (Hanner et al. 1997; Hayward et al. 2000; Wooden et al. 1999; Crovisier et al. 1997, 2000). The $10-\mu \mathrm{m}$ spectra exhibit several discrete peaks, which have been attributed to the presence of at least five different Mg-rich silicates in cometary dust: amorphous pyroxene, amorphous olivine, crystalline ortho-pyroxene, crystalline clino-pyroxene, and crystalline olivine (Wooden et al. 1999). ISO observations of the full $2-45 \mu \mathrm{m}$ spectra of comet Hale-Bopp showed several broad emission features longward $16 \mu \mathrm{m}$, all attributed to bending mode vibrations of Mg-rich crystalline olivine (Crovisier et al. 1997, 2000; Brucato et al. 1999a; Colangeli et al. 1999).

All interpretations of Hale-Bopp spectra agree on the presence of olivines and pyroxenes in both amorphous and crystalline form. However, they differ quantitatively. A summary of inferred relative abundances is given in Table 1. The relative mass fraction of silicates in crystalline form inferred from Hale-Bopp spectra varies from 30 to $90 \%$. Not included in Table 1 is the estimation made by Hayward et al. (2000) of $20 \%$ of crystalline silicates . As explained by Hanner (1999), it is difficult to determine accurately the relative abundances of minerals in cometary dust from their infrared spectra. With respect to other results, Wooden et al. (1999) suggest a much higher mass fraction of crystalline pyroxenes (Table 1). In contrast to other studies, they attribute the $9.3 \mu \mathrm{m}$ peak to crystalline pyroxene. In order to reproduce the relative strength of this feature observed at various heliocentric distances with a similar relative mass fraction of crystalline pyroxenes with respect to the other dust components, they argue that crystalline pyroxenes must be cooler than other silicate components, hence the different result. As discussed by Wooden et al., this requires that pyroxene crystals are separate particles in the Hale-Bopp's coma. This doesn't fit with current views of cometary dust as porous aggregate particles mixing silicates and carbonaceous material, and might deserve further studies (see the discussion of Hayward et al. 2000).

$10-\mu \mathrm{m}$ spectra obtained in other long-period comets indicate a silicate mineralogy in these comets similar to that in comet Hale-Bopp (Wooden et al. 1999). However, significant spectral differences are seen between comets. Colangeli et al. $(1995,1996)$ attribute these differences to variations in the relative amounts of the various silicates or in the $\mathrm{Mg} / \mathrm{Fe}$ ratio. Hayward et al. (2000) argue that they 
Table 1. Relative mass fractions of silicates in comet C/1995 O1 (Hale-Bopp).

\begin{tabular}{lccccccl}
\hline Date & $r_{\mathrm{h}}[\mathrm{AU}]$ & Cry Ol & Cry Pyr & Am Ol & Am Pyr & Cry Tot $^{a}$ & Reference \\
\hline 7 Oct. 1996 & 2.8 & 0.22 & 0.08 & $-^{b}$ & 0.72 & 0.30 & Crovisier et al. (2000) \\
& & 0.50 & $-^{b}$ & 0.10 & 0.40 & 0.50 & Colangeli et al. $(1999)^{c}$ \\
11 Apr. 1997 & \multirow{2}{*}{0.93} & 0.33 & 0.20 & 0.31 & 0.13 & 0.54 & Wooden et al. (1999) \\
& & 0.08 & $0.80^{d}$ & 0.08 & 0.03 & 0.88 & Wooden et al. (1999) \\
28 Dec. 1997 & 3.9 & 0.22 & 0.14 & $-^{b}$ & 0.64 & 0.56 & Crovisier et al. $(2000)$ \\
\hline
\end{tabular}

${ }^{a}$ Relative mass fraction of silicates in crystalline form, to be directly compared to model calculations of $C_{\mathrm{c}}$.

${ }^{b}$ Silicate component not considered in the fit of the spectrum.

${ }^{c}$ Spectral fit includes amorphous carbon. Silicate mass fractions have been here renormalized.

${ }^{d}$ Sum of ortho- and clino-pyroxene contributions. Ortho-pyroxene contribution is dominant.

${ }^{e}$ In contrast to other spectral analyses, crystalline pyroxenes are assumed to be cooler than other silicates.

might rather reflect variations in the dust size distribution and temperature.

Data on short-period comets, presumably formed in the Kuiper Belt, are very sparse. They usually exhibit weak and broad featureless $10 \mu \mathrm{m}$ emission, presumably due to their deficiency in small particles (Hanner et al. 1994). However, crystalline olivines might be present in short-period comets as well, as suggested by the ISO spectrum of comet $103 \mathrm{P} /$ Hartley 2 which shows the presence of a peak at $11.3 \mu \mathrm{m}$ (Crovisier et al. 1999, 2000).

\section{Model}

\subsection{Outlines}

The goal of our modelling is to investigate whether crystalline silicates formed in the inner warm regions of the solar nebula can be efficiently transported by turbulent diffusion to the outermost cold regions where comets presumably formed. This model treats both the amorphousto-crystalline phase transition of small silicate particles and their diffusive transport throughout the nebula as a function of time.

We use a simplistic approach in which only two dust components are considered, regardless of their mineralogy: amorphous and crystalline silicates. The amorphous-tocrystalline transition by thermal heating can be treated thanks to recent laboratory measurements (Sect. 3.3).

The model solves time-dependent equations which describe the diffusive transport of small dust particles in a turbulent accretion disk (Morfill \& Völk 1984). Defining $\rho$ as the mass density of the disk (dominated by $\mathrm{H}_{2}$ and $\mathrm{He}$ ), and $\rho_{k}$ as the mass density of dust particles of chemical index $k$, we have:

$$
\begin{aligned}
& \frac{\partial \rho_{k}}{\partial t}+\operatorname{div}\left(\boldsymbol{v} \rho_{k}\right)=\operatorname{div}\left[\kappa \rho \operatorname{grad}\left(\frac{\rho_{k}}{\rho}\right)\right]+Q_{k} \\
& \frac{\partial \rho}{\partial t}+\operatorname{div}(\boldsymbol{v} \rho)=0 .
\end{aligned}
$$

The second term in the left part of Eqs. (1) and (2) is the advection term describing inward mass transport towards the central object at the velocity $\boldsymbol{v}$. We assume that the dust particles are well mixed with the gas
(Dubrulle et al. 1995), so that their radial velocity $v_{\mathrm{r}}$ is the same as that of the gas. In the case of a thin Keplerian disk, $v_{\mathrm{r}}$ is equal to $-3 \nu_{\mathrm{t}} / 2 r$, where $\nu_{\mathrm{t}}$ is the turbulent viscosity and $r$ is the radial distance. The effect of the turbulence in the solar nebula is to smooth out any concentration gradient. This diffusion is represented by the first term in the right part of Eq. (1): the turbulent diffusivity $\kappa$ is set to the ratio of turbulent viscosity $\nu_{\mathrm{t}}$ to the Prandtl number $P_{\mathrm{r}}$. For reasons discussed in Hersant et al. (2001), $P_{\mathrm{r}}$ is taken equal to $0.7 . Q_{k}$ is a source or sink term which describes local production and destruction processes. Equation (2) is the equation of continuity of the total mass flow.

Using Eqs. (1) and (2), it can be shown that, in the midplane of the disk, the relative concentration $C_{k}=\rho_{k} / \rho$ follows the diffusion equation:

$\frac{\partial C_{k}}{\partial t}+v_{\mathrm{r}} \frac{\partial C_{k}}{\partial r}=\frac{1}{\Sigma r} \frac{\partial}{\partial r}\left(r \Sigma \kappa \frac{\partial C_{k}}{\partial r}\right)+\frac{Q_{k}}{\rho}$,

where $\Sigma$ is the surface density of the disk.

The annealing process of amorphous silicates and the diffusive transport of crystalline silicates in the outer regions of the solar nebula can be investigated by solving two diffusion equations which are coupled by source and sink terms:

$$
\begin{aligned}
& \frac{\partial C_{\mathrm{a}}}{\partial t}+v_{\mathrm{r}} \frac{\partial C_{\mathrm{a}}}{\partial r}=\frac{1}{\Sigma r} \frac{\partial}{\partial r}\left(r \sum \kappa \frac{\partial C_{\mathrm{a}}}{\partial r}\right)-\frac{Q}{\rho}, \\
& \frac{\partial C_{\mathrm{c}}}{\partial t}+v_{\mathrm{r}} \frac{\partial C_{\mathrm{c}}}{\partial r}=\frac{1}{\Sigma r} \frac{\partial}{\partial r}\left(r \sum \kappa \frac{\partial C_{\mathrm{c}}}{\partial r}\right)+\frac{Q}{\rho} .
\end{aligned}
$$

The indices "a" and "c" refer to amorphous and crystalline silicates, respectively, and $Q$ is the coupling term representing the annealing process. If $t_{\mathrm{ann}}$ is the characteristic timescale for annealing (Sect. 3.3), then $Q=C_{\mathrm{a}} \rho / t_{\mathrm{ann}}$ and we have:

$$
\begin{aligned}
& \frac{\partial C_{\mathrm{a}}}{\partial t}+v_{\mathrm{r}} \frac{\partial C_{\mathrm{a}}}{\partial r}=\frac{1}{\Sigma r} \frac{\partial}{\partial r}\left(r \sum \kappa \frac{\partial C_{\mathrm{a}}}{\partial r}\right)-\frac{C_{\mathrm{a}}}{t_{\mathrm{ann}}}, \\
& \frac{\partial C_{\mathrm{c}}}{\partial t}+v_{\mathrm{r}} \frac{\partial C_{\mathrm{c}}}{\partial r}=\frac{1}{\Sigma r} \frac{\partial}{\partial r}\left(r \sum \kappa \frac{\partial C_{\mathrm{c}}}{\partial r}\right)+\frac{C_{\mathrm{a}}}{t_{\mathrm{ann}}} .
\end{aligned}
$$

Equations (6) and (7) do not include sublimation and condensation terms. Sublimation is taken into account in 
the boundary conditions by setting $C_{\mathrm{a}}=C_{\mathrm{c}}=0$ at the distance $r$ where the temperature of the nebula is high enough to allow efficient sublimation (Sects. 3.2 and 3.3). We do not consider the re-formation of silicates which subsequently occurs as the hot gas is cooling down (see the discussion in Sect. 4).

Solving Eqs. (6) and (7) requires an evolutionary solar nebula model giving surface density and temperature radial profiles in the midplane of the disk as a function of time.

\subsection{Solar nebula model}

We sum up here the solar nebula model that we use, and which is described in length by Hersant et al. (2001). The temporal evolution of the solar nebula is modelled by a sequence of stationary solutions provided by the 2-D accretion disk model of Huré (2000), where the vertical stratification of the physical quantities is explicitly calculated. In a $2-\mathrm{D}$ model, the midplane temperature, pressure, and volumetric density are generally slightly higher than in a 1-D model where a vertically homogeneous disk is assumed (Huré \& Galliano 2001). This model uses the well known $\alpha$ prescription of Shakura \& Sunyaev (1973), which permits modellers to parameterize the effects of small scale turbulence. The prescription consists of writing the turbulent viscosity as $\nu_{\mathrm{t}}=\alpha C_{\mathrm{s}}^{2} / \Omega$, where $C_{\mathrm{s}}$ is the sound velocity and $\Omega$ is the Keplerian angular velocity. $\alpha$ is a non-dimensional parameter whose value depends on the nature of the turbulence and is usually taken, for simplicity, as constant with respect to the heliocentric distance in the disk and time.

The 2-D model is calculated for a given accretion rate $\dot{M}$ which governs the inflow of matter from the disk onto the proto-Sun. As the disk evolves and cools down, the turbulence is less and less efficient, and this accretion rate $\dot{M}$ diminishes. The evolutionary model begins when the formation of the Sun is almost complete. The subsequent evolution of the nebula is obtained by calculating 2-D models for a temporal sequence of accretion rates following the prescription of Makalkin \& Dorofeyeva (1991). These authors have shown that the evolution of $\dot{M}$ as a function of time can be described by a simple power law which depends upon the accretion rate and the outer radius of the nebula $R_{\mathrm{D} 0}$ at $t=0$ according to:

$\dot{M}(t)=\dot{M}_{0}\left(1+t / t_{0}\right)^{-1.5}$

with

$t_{0}=\frac{R_{\mathrm{D} 0}^{2}}{3 \nu_{\mathrm{t}}\left(R_{\mathrm{D} 0}\right)}$,

where $\nu_{\mathrm{t}}\left(R_{\mathrm{D} 0}\right)$ is the coefficient for turbulent viscosity at the initial outer radius of the nebula. Such evolution agrees with the numerical evolutionary models of Ruden \& Lin (1986) or Ruden \& Pollack (1991). It is also consistent with the decrease of the mass accretion rate with increasing age observed in T Tauri stars (Hartmann et al. 1998).
Table 2. Solar nebula models.

\begin{tabular}{lccc}
\hline Type & $\alpha$ & $\begin{array}{c}\dot{M}_{0} \\
{\left[M_{\odot \mathrm{yr}^{-1}}\right]}\end{array}$ & $\begin{array}{c}R_{\mathrm{D} 0} \\
{[\mathrm{AU}]}\end{array}$ \\
\hline cold & $2 \times 10^{-2}$ & $4.0 \times 10^{-6}$ & 27.0 \\
nominal & $9 \times 10^{-3}$ & $5.0 \times 10^{-6}$ & 17.0 \\
warm & $8 \times 10^{-3}$ & $9.8 \times 10^{-6}$ & 12.8 \\
\hline
\end{tabular}

The model takes also into account that, under the action of turbulence, the nebula spreads out viscously with time. It is assumed that the evolution of its outer radius $R_{\mathrm{D}}$ follows:

$\frac{\mathrm{d} R_{\mathrm{D}}}{\mathrm{d} t}=\frac{3}{2} \frac{\nu_{\mathrm{t}}}{R_{\mathrm{D}}}$

The model input parameters are finally the initial radius $R_{\mathrm{D} 0}$ and accretion rate $\dot{M}_{0}$ of the nebula, and $\alpha$.

With this solar nebula model, Hersant et al. studied the radial distribution and time evolution of the deuterium enrichment in water in the solar nebula due to isotopic exchange with $\mathrm{H}_{2}$ and diffusive turbulent transport. Assuming that the highly D-enriched component measured in LL3 meteorites $\left(\mathrm{D} / \mathrm{H}=(73 \pm 12) \times 10^{-5}\right)$ is representative of the grains in the presolar cloud (Drouart et al. 1999; Mousis et al. 2000), Hersant et al. were able to explain the $\mathrm{D} / \mathrm{H}$ value of $\sim 30 \times 10^{-5}$ measured in cometary water as due to some mixing between water vapor reprocessed in the inner regions of the solar nebula by isotopic exchange with $\mathrm{H}_{2}$, and the unprocessed component of its outer regions. Hersant et al. (2001) showed that the three parameters defining the nebula can be constrained by the deuterium enrichments measured in LL3 meteorites, comets, proto-Uranian and proto-Neptunian ices, taking into account a few other physical constraints, namely: 1) the mass of the nebula cannot exceed $0.3 M_{\odot}$ in order to maintain the stability of the disk (Shu et al. 1990); 2) the angular momentum must have been transported outward to Neptune by turbulence in $2.5 \times 10^{5} \mathrm{yr}$; 3 ) the initial temperature of the nebula was higher than $1000 \mathrm{~K}$ inside $3 \mathrm{AU}$ to secure the crystallization of silicates in the inner regions.

Hersant et al. concluded that $\dot{M}_{0}$ was in between $2.2 \times 10^{-6}$ and $10^{-5} M_{\odot} \mathrm{yr}^{-1}, R_{\mathrm{D} 0}$ between 12.8 and $39 \mathrm{AU}$, and $\alpha$ between 0.006 and 0.02 . For the purpose of this paper in which the temperature of the nebula is crucial for determining where and when the conversion of amorphous silicates into the crystalline variety occurred, they selected among all nebulae fitting observational constraints one of the coldest and one of the hottest nebulae, named cold nebula and warm nebula, respectively. Their parameters are given in Table 2. They also selected a nebula intermediate between the cold and warm nebulae, called nominal nebula (Table 2). Radial profiles of $\Sigma$ and $T$ as a function of time are shown in Fig. 1 for these three nebulae. Adiabats $(P, T)$ of the nominal solar nebula at various epochs are shown in Fig. 2. 


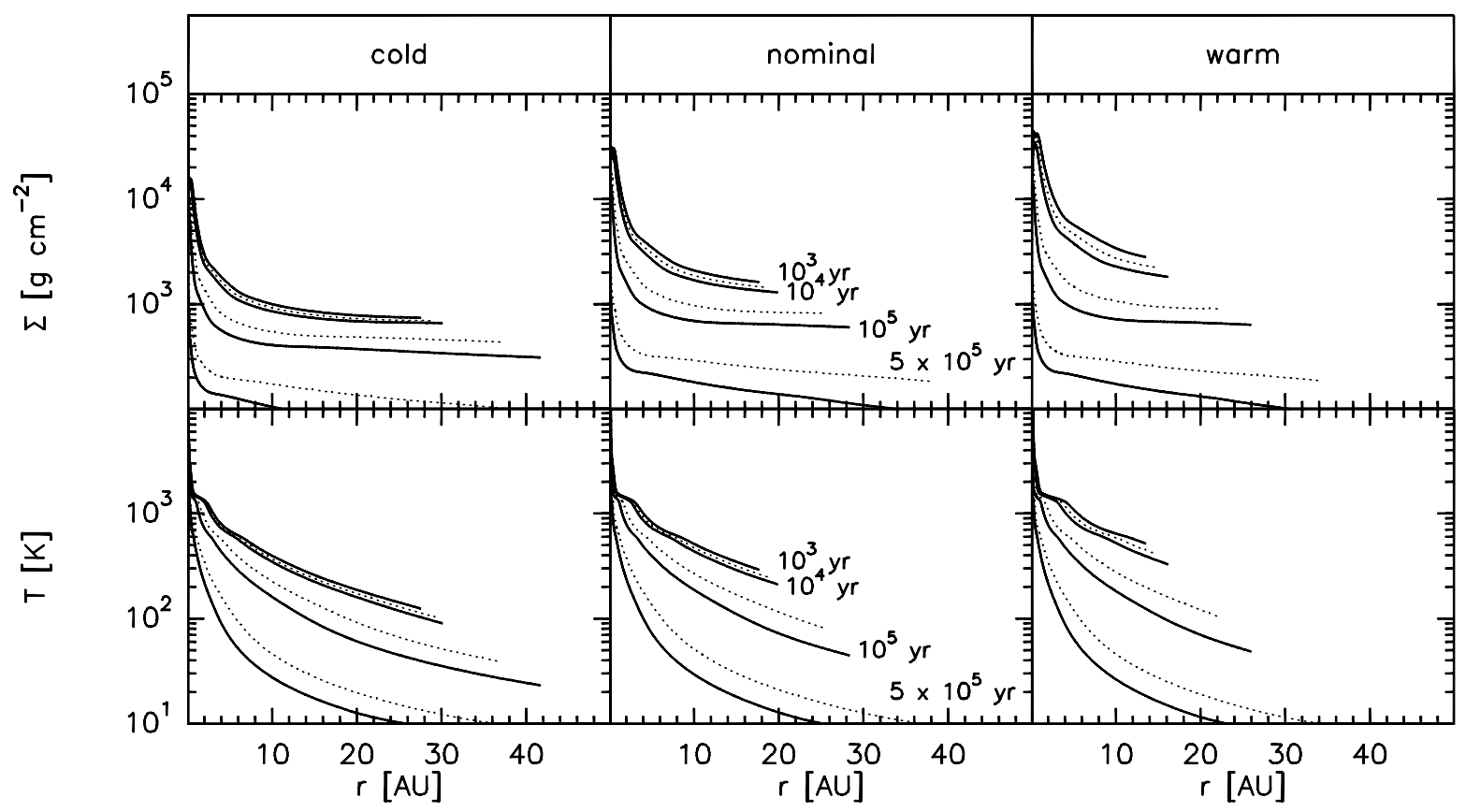

Fig. 1. Surface density $(\Sigma)$ and temperature $(T)$ radial profiles as a function of time for the three nebulae defined in Table 2 . Plain lines are for times $10^{3}, 10^{4}, 10^{5}$, and $10^{6}$ years. Dotted lines are for times $5 \times 10^{3}, 5 \times 10^{4}$, and $5 \times 10^{5}$ years.

\subsection{Silicates crystallization and sublimation}

Solving Eqs. (6) and (7) requires, in addition to a solar nebula model, data on silicates crystallization and sublimation.

The thermally induced amorphous-to-crystalline transition has been studied both theoretically (see Gail 1998 and references therein) and in the laboratory (e.g. Ashworth et al. 1984; Nuth \& Donn 1982; Hallenbeck et al. 1998; Brucato et al. 1999b; Fabian et al. 2000). The crystallization process corresponds, on a microscopic scale, to the movement of oxygen and silicon atoms to new locations characterized by deeper local energy minima. The characteristic timescale for the amorphous-crystalline transition can be expressed as:

$t_{\text {ann }}=\nu^{-1} \mathrm{e}^{E_{\mathrm{a}} / k T}$,

where $\nu$ is a characteristic vibrational frequency and $E_{\mathrm{a}}$ acts as an activation energy (Lenzuni et al. 1995). In experimental determinations of $E_{\mathrm{a}}, \nu$ is set to $2-2.5 \times 10^{13} \mathrm{~s}^{-1}$, the average value between the frequencies of the stretching and bending modes of the $\mathrm{SiO}_{4}$ tetrahedron (Lenzuni et al. 1995; Gail 1998). From thermal annealing experiments made at $\sim 1100$ and $1300 \mathrm{~K}$ on $\sim 20$-nm sized smoke particles, Brucato et al. (1999b) estimated an activation temperature $E_{\mathrm{a}} / k$ of $47500 \mathrm{~K}$ for amorphous Mg-rich pyroxenes (hereafter referred to as $\mathrm{MgSiO}_{3}$, enstatite). Brucato et al. (1999b) derived a temperature of $45500 \mathrm{~K}$ from the annealing experiment of Hallenbeck et al. (1998). Experiments made at $1000 \mathrm{~K}$ by Fabian et al. (2000) on nanometre-sized smoke particles of Mg-rich pyroxene and $\mathrm{Mg}$-rich olivine $\left(\mathrm{Mg}_{2} \mathrm{SiO}_{4}\right.$, forsterite) give activation temperatures of 42040 and
$39100 \mathrm{~K}$, respectively. The difference in the measured activation energies for Mg-rich pyroxene between Brucato et al. (1999b) and Fabian et al. (2000) comes from a different definition of the annealing time. Both studies monitored the evolution of IR spectra of silicates undergoing annealing. While Fabian et al. define the annealing time as the time when the first crystalline IR features appear, Brucato et al. define it as the time when crystalline IR features no longer evolve. Between these two stages, Hallenbeck et al. (1998) observed a stall phase in the spectra before the features sharpened due to the crystal becoming more ordered.

Experiments revealed a significant distinction in the amorphous-to-crystalline transition between micrometresized glass and smoke particles (Fabian et al. 2000). This is something interesting to consider, especially given the large size of interstellar dust (typically $\sim 0.1 \mu \mathrm{m}$ ) compared to that of the laboratory smokes. First, the timescale at $1000 \mathrm{~K}$ for crystallization of $\mathrm{MgSiO}_{3}$ glass particles exceeds that of $\mathrm{MgSiO}_{3}$ smoke particles. In addition, the activation temperature determined for these micrometre particles with $\nu=2 \times 10^{13} \mathrm{~s}^{-1}$ decreases with increasing annealing temperature (Fabian et al. 2000). We performed a least-square fit to the annealing times measured for temperatures between $1030 \mathrm{~K}$ and $1121 \mathrm{~K}$ (median values of Table 3 in Fabian et al. 2000), which shows that the measurements can be fitted by an exponential law (Eq. (11)) with $\nu=4.7 \times 10^{24} \mathrm{~s}^{-1}$ and $E_{\mathrm{a}} / k=70060 \mathrm{~K}$ (note that the uncertainties on $\nu$ and $E_{\mathrm{a}} / k$ are large: respectively at least \pm 2 orders of magnitude and $\pm 5000 \mathrm{~K}$, taking the range of estimated annealing times as the uncertainty on the measurements). Although this law may not apply over a large temperature range, it permits us 
Table 3. Laws for annealing timescales.

\begin{tabular}{lllll}
\hline Law $^{a}$ & Compound & $\begin{array}{l}\nu \\
{\left[\mathrm{s}^{-1}\right]}\end{array}$ & $\begin{array}{l}E_{\mathrm{a}} / k \\
{[\mathrm{~K}]}\end{array}$ & $\begin{array}{c}t_{\text {ann }} \\
1000 \mathrm{~K}[\mathrm{~s}]\end{array}$ \\
\hline$(1)$ & $\mathrm{Mg}_{2} \mathrm{SiO}_{4}$ smoke & $2 \times 10^{13}$ & 39100 & $4.8 \times 10^{4}$ \\
$(2)$ & $\mathrm{MgSiO}_{3}$ smoke & $2 \times 10^{13}$ & 42040 & $9.1 \times 10^{4}$ \\
$(3)$ & $\mathrm{MgSiO}_{3}$ smoke & $2.5 \times 10^{13}$ & 47500 & $1.7 \times 10^{7}$ \\
$(4)$ & $\mathrm{MgSiO}_{3}$ glass & $5 \times 10^{24}$ & 70000 & $5.0 \times 10^{5}$ \\
$(5)$ & $\mathrm{MgSiO}_{3}$ glass & $2.5 \times 10^{26}$ & 70000 & $1.0 \times 10^{4}$ \\
\hline
\end{tabular}

a Timescale laws for crystallization parameterized by $\nu$ and $E_{\mathrm{a}} / k$ : (1) $\mathrm{Mg}_{2} \mathrm{SiO}_{4}$ smoke, Fabian et al. (2000); (2) $\mathrm{MgSiO}_{3}$ smoke, Fabian et al. (2000); (3) $\mathrm{MgSiO}_{3}$ smoke, Brucato et al. (1999b); (4) $\mathrm{MgSiO}_{3}$ glass, Fabian et al. (2000), see text; (5) $\mathrm{MgSiO}_{3}, 0.1 \mu \mathrm{m}$ glass, see text.

to investigate larger timescales for crystallization with respect to those derived for nanometre smoke particles.

Table 3 gives the laws for $t_{\text {ann }}$ considered in this paper and $t_{\mathrm{ann}}$ values at $T=1000 \mathrm{~K}$. The laws (1) to (3) come from the mentioned experiments on smoke particles. Law numbered (4) is that derived in the paragraph above, from measurements on micrometre grains. The law numbered $(5)\left(\nu=2.5 \times 10^{26} \mathrm{~s}^{-1}\right.$ and $\left.E_{\mathrm{a}} / k=70000 \mathrm{~K}\right)$ is possibly more appropriate for $0.1 \mu \mathrm{m}$ sized particles. It was derived from law (4), assuming the linear grain size dependence suggested by annealing experiments made on silicate glassy sheets (Fabian et al. 2000).

The annealing timescales we use are those determined for nearly pure magnesium silicates. It can be objected that these measurements are not fully appropriate for our purpose, since amorphous silicates in the protosolar cloud contained some iron. Demyk et al. (1999) studied the infrared signatures of the silicates around two massive protostellar objects and showed that they can be well reproduced with $\mathrm{Mg}-\mathrm{Fe}$ pyroxenes amorphous grains with a $\mathrm{Fe} / \mathrm{Mg}$ ratio of about 0.5 . It would be thus interesting to use annealing timescales characteristic of such compounds. Annealing experiments have been made for pure iron-silicate and magnesium-iron silicates (Hallenbeck et al. 1998), but the activation temperatures required for our model are not available. Hallenbeck et al. (1998) found that annealing timescales are much larger for iron silicates than for magnesium silicates. On the other hand, the evolution of mixed magnesium-iron silicates resembles that of magnesium silicate samples (Hallenbeck et al. 1998).

The stability of Mg-rich silicates against vaporization has been studied by Duschl et al. (1996). It is shown that silicates decompose in a near equilibrium process with the gas phase and gradually disappear over a finite temperature interval of $\sim 300 \mathrm{~K}$. We have considered the $\left(P_{\text {sat }}, T\right)$ equilibrium curve with $f=0.9$ given in Fig. 4 of this paper, which corresponds to the situation where a fraction $f=0.9$ of the $\mathrm{Si}$ is still bound into grains. This curve is plotted in Fig. 2 over the $(P, T)$ adiabat of the solar nebula. The last grains disappear at a temperature $\sim 100 \mathrm{~K}$ higher (Duschl et al. 1996). Taking the $\left(P_{\text {sat }}, T\right)$

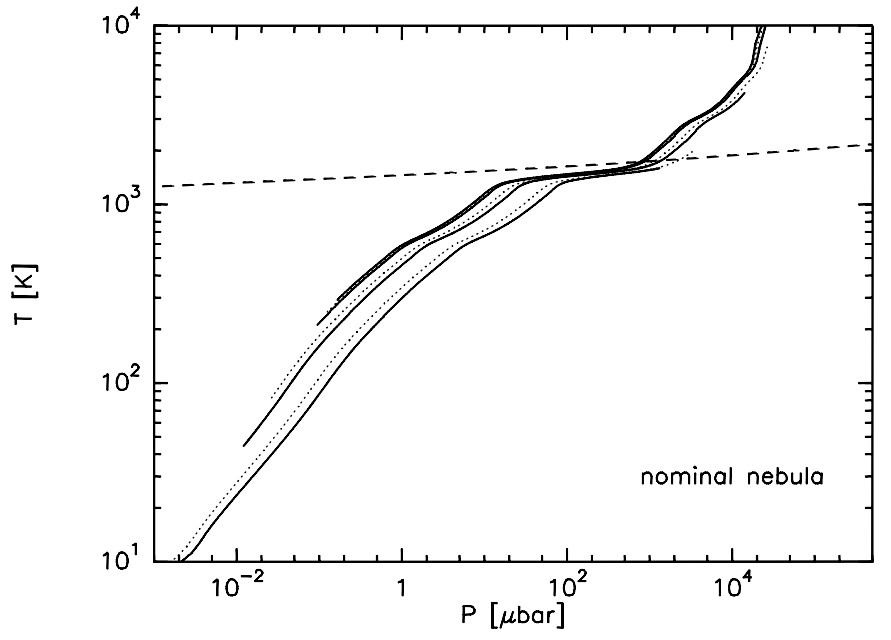

Fig. 2. Adiabat $(P, T)$ of the nominal nebula as a function of time. Curves from top to bottom correspond to increasing times. Plain lines are for times $10^{3}, 10^{4}, 10^{5}$, and $10^{6}$ years. Dotted lines are for times $5 \times 10^{3}, 5 \times 10^{4}$, and $5 \times 10^{5}$ years. The dashed line shows the equilibrium curve against vaporization of silicates taken from Duschl et al. (1996). This equilibrium curve intersects the nebula adiabat at $r \sim 0.7,0.6,0.4,0.3$, and $0.1 \mathrm{AU}$ for times of $10^{3}, 10^{4}, 5 \times 10^{4}, 10^{5}$, and $5 \times 10^{5}$ years, respectively.

equilibrium curve with $f=0$ that corresponds to the full disappearance of silicate grains would not change significantly our results, keeping in mind that sublimation curves depend somewhat on the silicate composition.

\subsection{Integration of the diffusion equations and boundary conditions}

Numerical integration of the coupled partial differential Eqs. (6) and (7) requires initial conditions and boundary conditions at the inner $r_{0}$ and outer $R_{\mathrm{D}}$ radii at any time. This is a classical initial value problem which can be solved by finite differencing, given a $r$-grid and time step.

Equations (6) and (7) mix advective and diffusion terms, each of them requiring a specific differencing scheme to avoid numerical dissipation and stability problems. Therefore, we used the "operator splitting" method and computed separately, and successively within one time step, the time evolution of $C_{\mathrm{a}}$ and $C_{\mathrm{c}}$ due to the advective and diffusion terms. The time evolutions resulting from the chemical and diffusion terms were treated together.

For the transport term, we used the explicit spatial differencing scheme suggested by Hawley et al. (1984) (Sect. II.c.ii) which mixes the second-order (in space) averaging scheme of Wilson (1978) and the first-order upwind scheme. For treating the diffusion, we used the CrankNicholson differencing scheme which is second-order in both space and time (see Drouart et al. 1999).

The $r$-grid has been defined between $r_{0}=0.1 \mathrm{AU}$ and $R_{\mathrm{D}}$ with logarithmic steps such that $r_{i+1}=r_{i} \times 1.03$. The time step has been set to $\min \left[t_{\mathrm{ann}}\left(r_{0}\right) / 100,50 \mathrm{yr}\right]$. 
For minimizing the computer time, $t_{\text {ann }}$ values less than $50 \mathrm{yr}(5 \mathrm{yr}$ for the cold nebula) were set to $50 \mathrm{yr}$ ( $5 \mathrm{yr}$ for the cold nebula).

As initial condition, we took $C_{\mathrm{a}}=C t$ and $C_{\mathrm{c}}=0$ at any $r$, where $C t$ is a constant setting the relative mass concentration of silicates with respect to $\mathrm{H}_{2}$. Since Eqs. (6) and (7) are linear in $C t$ and we are interested in the relative fraction of crystalline versus amorphous silicates, we took $C t=1$. $C_{\mathrm{a}}$ and $C_{\mathrm{c}}$ then provides directly the relative mass fractions of amorphous and crystalline silicates, respectively. With this initial condition, we assume that the silicates are only in amorphous form throughout the nebula at $t=0$. Due to hot temperatures at small $r$, the initial concentrations are in fact determined by the thermal radial profile of the nebula at $t=0$. Figure 3 shows that silicates are essentially amorphous at distances larger than $10 \mathrm{AU}$ for the considered nebulae. These initial conditions are valid, providing that the grains outside $10 \mathrm{AU}$ were not thermally processed in the preceding stages of solar nebula formation and evolution. As discussed in Sects. 1 and 4.1, silicates suffered little processing during the collapse phase (Chick \& Cassen 1997).

The boundary spatial condition $\frac{\partial C_{\mathrm{a}(\mathrm{c})}}{\partial r}=0$ was taken at $r=R_{\mathrm{D}}$. This implies that we neglect radial influx of material from the outside. When the sublimation boundary $r_{\text {sub }}$ extends farther than $r_{0}=0.1 \mathrm{AU}$, the inner spatial boundary condition is $C_{\mathrm{a}}=C_{\mathrm{c}}=0$ at $r \leq r_{\text {sub. }}$. When this is not the case (for the considered nebulae, this happens only at $t>5 \times 10^{5} \mathrm{yr}$, Fig. 3 ), we assumed, for simplicity, $\frac{\partial C_{\mathrm{a}(\mathrm{c})}}{\partial r}=0$.

\section{Results and discussion}

The temporal and radial evolution of the relative mass fraction of crystalline silicates $C_{\mathrm{c}}$ is shown in Fig. 3, for the three considered nebulae. In Fig. 4 is plotted the mass fraction of crystalline silicates integrated over the whole nebula $C_{\mathrm{c}}^{\text {int }}$ as a function of time:

$C_{\mathrm{c}}^{\mathrm{int}}=\frac{\int_{r_{0}}^{R_{\mathrm{D}}} 2 \pi r C_{\mathrm{c}} \Sigma \mathrm{d} r}{\int_{r_{0}}^{R_{\mathrm{D}}} 2 \pi r \Sigma \mathrm{d} r}$.

The calculations shown in Figs. 3-4 refer to the annealing timescales $t_{\text {ann }}$ computed with the law (1) at the top of Table 3, namely $\nu=2 \times 10^{13} \mathrm{~s}^{-1}$ and $E_{\mathrm{a}} / k=39100 \mathrm{~K}$ (Fabian et al. 2000). The calculations made with the other laws given in Table 3 provide qualitatively the same results.

At time slightly above $0 \mathrm{yr}$, silicate grains are vaporized or are in crystalline form within some distance which depends on the initial thermal structure of the nebula. This distance, that we call the crystalline front, is $\sim 5$ to $7 \mathrm{AU}$ for the considered nebulae. As time goes on, amorphous silicates, present in the outer regions, are transported towards the inner regions by advection and turbulent diffusion, and then thermally annealed. This radial inward flux of amorphous material inside the region where crystallization takes place balances the loss of crystalline silicates by advective transport within the evaporation zone. As shown in Fig. 4, the integrated mass fraction of crystalline silicates is maintained to a value that slightly exceeds the value acquired at $t \approx 0$ up to times $\sim 2 \times 10^{4}$ yr due to on-going crystallization. When mass input from crystallization no longer balances loss by evaporation, the integrated mass fraction of crystalline silicates then decreases to reach a plateau value. The ratio between this long term plateau value and the crystalline mass fraction at $t \approx 0$ is more than $25 \%$ for the nebulae considered here (Fig. 4). This shows that turbulent diffusion of both amorphous and crystalline silicates is an efficient process for maintaining a high proportion of crystalline silicates in the solar nebula, as it is cooling down.

The effects of turbulent diffusion are seen on the radial profiles of $C_{\mathrm{c}}$ shown in Figs. 3A-C. Crystalline silicates are progressively mixed with the amorphous grains present in the outer regions, so that $C_{\mathrm{c}}$ continuously increases in the outer regions to finally reach a plateau equal to the $C_{\mathrm{c}}^{\mathrm{int}}$ plateau value mentioned above. In turn, the crystalline front moves towards the Sun, as the nebula cools with time.

The characteristic timescale for radial mixing is approximately given by the accretion rate timescale $t_{0}$ defined by Eq. (9) and equal to $\sim 4.0 \times 10^{4}, 3 \times 10^{4}$ and $1.6 \times 10^{4} \mathrm{yr}$ for the cold, nominal and warm nebulae, respectively (Hersant et al. 2001). Figure 3 shows that, indeed, the plateau is reached faster for the warm nebula than for the nominal nebula, and faster for the nominal nebula than for the cold nebula. For example, at $t=10^{4} \mathrm{yr}$, the value of $C_{\mathrm{c}}$ at $r=15 \mathrm{AU}$ is $\sim 80 \%$ the plateau value for the warm nebula, while, at the outermost distances of the cold nebula, the silicates are still essentially amorphous. At times larger than $\sim 5-10 \times 10^{4} \mathrm{yr}$, the mass fraction of crystalline silicates at $r>10 \mathrm{AU}$ no longer evolves for the three nebulae considered here. The timescale for radial mixing is governed by the diffusion timescale $\tau_{\text {diff }}=r^{2} / \nu_{\mathrm{t}}$, which increases rapidly with increasing distance, and also increases significantly with increasing time, due to decreasing temperature and disk thickness with time (Fig. 5).

Table 4. Crystalline mass fraction in the outer solar nebula at infinite time.

\begin{tabular}{llccc}
\hline Law $^{a}$ & Compound & \multicolumn{3}{c}{$C_{\mathrm{c}}$} \\
& & \multicolumn{3}{c}{ Solar nebula } \\
\cline { 3 - 5 } & & cold & nominal & warm \\
\hline$(1)$ & $\mathrm{Mg}_{2} \mathrm{SiO}_{4}$ smoke & 0.022 & 0.123 & 0.58 \\
$(2)$ & $\mathrm{MgSiO}_{3}$ smoke & 0.018 & 0.094 & 0.44 \\
$(3)$ & $\mathrm{MgSiO}_{3}$ smoke & 0.013 & 0.064 & 0.29 \\
$(4)$ & $\mathrm{MgSiO}_{3}$ glass & 0.013 & 0.066 & 0.30 \\
$(5)$ & $\mathrm{MgSiO}_{3}, 0.1 \mu \mathrm{m}$ glass & 0.015 & 0.076 & 0.35 \\
\hline
\end{tabular}

${ }^{a}$ Timescale laws for silicate crystallization labelled according to Table 3. 

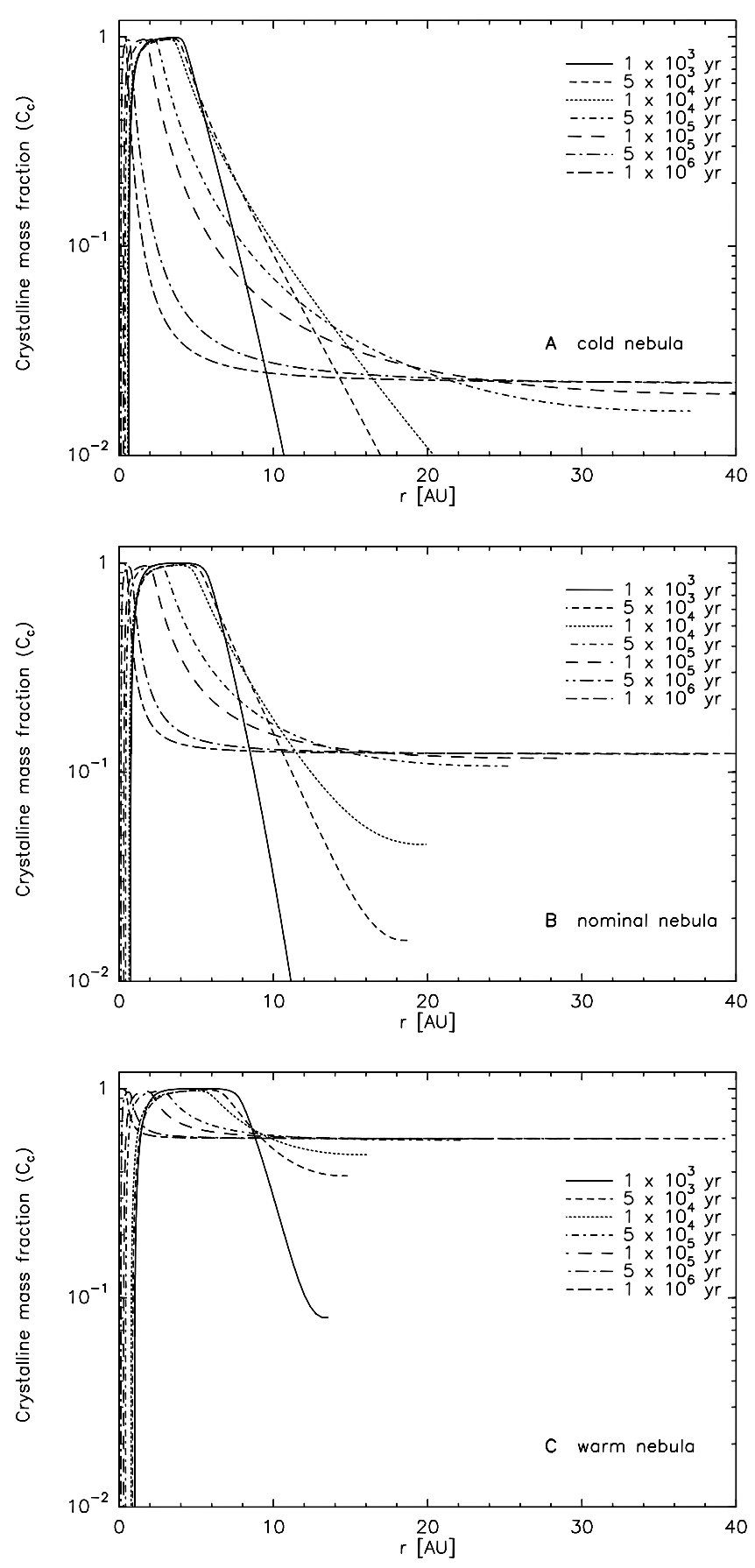

Fig. 3. Mass fraction of crystalline silicates as a function of radius and time in the solar nebula. Thermal annealing is modelled using law (1) of Table $3\left(\nu=2 \times 10^{13} \mathrm{~s}^{-1}\right.$ and $E_{\mathrm{a}} / k=39100 \mathrm{~K}$; Fabian et al. 2000). A) cold nebula. B) nominal nebula. C) warm nebula.

The mass fraction of crystalline silicates in the plateau is, as expected, strongly dependent on the thermal structure of the early nebula. The cold and warm nebulae considered here are extreme, so that the crystalline fraction varies from $\sim 0.02$ to 0.6 for the annealing timescales considered in the calculations shown in Fig. 3. Table 4 shows that, in contrast, the degree of crystallinity reached in the plateau is not very sensitive to the $t_{\text {ann }}$ law. Comparing

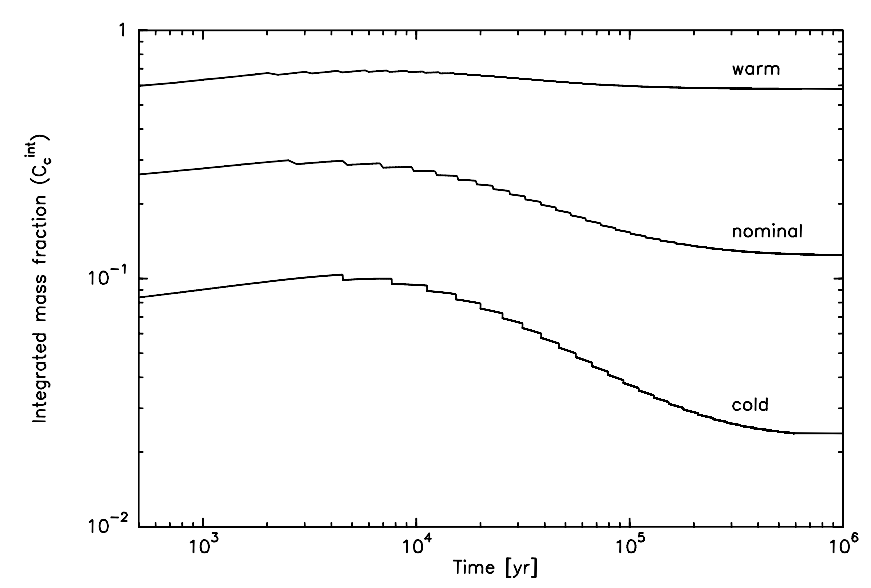

Fig. 4. Mass fraction of crystalline silicates integrated over the whole nebula $C_{\mathrm{c}}^{\text {int }}$ (Eq. (12)) as a function of time. Thermal annealing is modelled using law (1) of Table 3. Calculations for the three nebulae are shown.

results obtained for laws (1) and (2), the model predicts similar (within 30\%) degrees of crystallinity for olivine and pyroxene grains. The degree of crystallinity is lower for Mg-rich pyroxene grains than for olivine, due to their slightly higher activation temperature $E_{\mathrm{a}} / k$. The $t_{\text {ann }}$ laws used for modelling the annealing of $\mathrm{MgSiO}_{3}$ glass particles (laws (4) and (5)) provide results not very different (within 40\%) from those derived using experimental data on smoke $\mathrm{MgSiO}_{3}$ particles (law (2)).

\subsection{Formation of silicates from vapor phase}

The present work is based on the assumption that crystalline silicates were formed from the annealing of amorphous silicates moving radially in the disk equatorial plane towards the inner hot regions. Another mechanism for the formation of crystalline silicates may result from the recondensation of silicates. Silicates infalling onto the disk underwent heating in the collapsing presolar cloud or during the shock when arriving onto the disk. Chick \& Cassen (1997) have investigated the temperatures experienced by infalling interstellar grains during this early stage of Solar System formation and conclude that silicates evaporate within 1 AU. Both theoretical considerations and compositional constraints from meteorites argue that the thermal regime experienced in the midplane of the accretion disk was more severe and controlled the vaporization distances of refractory grains (Chick \& Cassen 1997). In the solar nebula models investigated here, the vaporization distance of silicates is $\sim 1 \mathrm{AU}$, a value less than the value $r_{\text {sub }} \sim 2$ AU required to explain the relative abundances of moderately volatile elements of carbonaceous meteorites (Cassen 1996, 2001).

We do not take into account in our model the condensation of silicates in the vapor phase which takes place near the Sun when the nebula cools down. This would require a kinetic treatment of the chemical surface reactions during grain growth for which data are lacking 
(Gail 1998; Gail \& Sedlmayr 1999), and would demand a coupling of the dust and gas diffusion equations. Should the condensation of silicates actually produce the crystalline form, as is expected, neglecting this process leads to an underestimation of the relative fraction of crystalline silicates with respect to the amorphous variety. Since the mass of material located within the sublimation region is much smaller than the mass located outside $(<10 \%$ for the selected nebulae; see Fig. 14 of Hersant et al. 2001), we believe that neglecting the contribution of crystalline silicates condensed from the vapor phase does not significantly affect our results. There are however presumptions that vapour phase condensates are present in cometary grains. A class of very porous interplanetary dust particles called $\mathrm{CP}$, and suspected to be of cometary origin, shows Mg-rich pyroxene crystals with whisker and platelet morphologies (Bradley et al. 1983). These micro-structures are expected to grow by condensation from a relatively lowpressure vapour phase.

\subsection{Silicates in meteorites}

Silicates in meteorites are all in crystalline form. However, crystalline silicates in meteorites were not necessarily produced by the annealing process investigated in the present work. Therefore, we cannot discuss the crystallinity of meteorites only in the context of our model, although there is no apparent conflict, since Fig. 3 shows that silicates within 3 AU remain essentially crystalline at times less than $10^{5} \mathrm{yr}$.

Most meteorites - stony, stony-iron and iron - record melting events related to the metamorphism and magmatism of at least, seventy small parent bodies (Scott 1991). In addition, a large number of asteroids seem differentiated. Although the mechanism that yielded this increase in temperature of the asteroidal parent body is still largely debated, no amorphous interstellar silicates present in the protosolar nebula would have survived such thermal stress linked to the planetesimal formation and evolution. Therefore, in the inner zone of the solar system, all solid bodies appear crystalline.

Among the classes of meteorites that could have preserved a partial record of the crystalline state of their amorphous silicate precursors, are the carbonaceous and the unequilibrated type 3 chondrites (especially the type LL3.0). These meteorites consists of chondrules and matrix.

Chondrules are 100 to $1000 \mu \mathrm{m}$ size spheroidal silicates, mainly made of olivine and pyroxene, which constitute the dominant fraction (up to 70\%) of chondrites. They are the products of partial melting of precursor silicates - possibly the amorphous interstellar dust with negligible contribution of sulphides, oxides and iron. Although the conditions of formation of chondrules are not totally understood, experimental simulations have allowed a precise reconstruction of their thermal history. (1) Their heating time during which sub-melting was taking place varied from $100 \mathrm{~s}$ (Tsuchiyama \& Nagahara 1981) to $20 \mathrm{~h}$ (Lofgren \& Russell 1988). (2) Essentially based on the distribution of the chemical elements between the different mineralogical phases coexisting in chondrules (as for example the $\mathrm{Fe} / \mathrm{Mg}$ partitioning coefficients) cooling rates were estimated to lie between 100 to $2000{ }^{\circ} \mathrm{C} / \mathrm{hr}$ (Radomsky \& Hewins 1990). (3) The range of heating temperature depends almost exclusively on the $\mathrm{Mg}-\mathrm{Al}-\mathrm{Si}$ chemical proportions of the chondrules and ranges from 1200 to $1700^{\circ} \mathrm{C}$. The occurrence of the three common textures (granular in Mg-rich olivine chondrules, pophyritic in Fe-rich olivine chondrules, radiating in pyroxene chondrules) are experimentally reproduced by heating the precursor silicates to near-liquidus temperature (1300$1800{ }^{\circ} \mathrm{C}$ ) but not higher (Hewins \& Radomsky 1990).

These short heating and cooling times associated with these high silicate melting temperatures are consistent with the idea that chondrules formed by transient heating phenomena (as those involved in electric discharges) in the dust-rich regions of the protosolar nebula. These conditions are not accounted for in the present model. It is clear that amorphous precursors of chondrules, if any, would not have survived through such a violent thermal history.

As far as the matrix is concerned, its origin is poorly documented mainly because of its very fine grain structure. The matrix is essentially composed of broken fragments of chondrules, of clay minerals and sub-micronic silicates and sulfides which are also found as relic grains in chondrules. Matrix lumps are sometimes enclosed in chondrule rims and therefore the formation of the matrix post-date the chondrule formation. The fact that amorphous mineral are extremely rare in the matrix cannot be taken as an evidence that the dust was entirely crystalline at the time and location of the matrix formation. Indeed, an intense circulation of water in the parent body meteorites is attested by the presence of low temperature phyllosilicates (such as smectite) essentially located in the matrix. For a given chemical composition, the formation rate of clay minerals is enhanced for amorphous compared to crystalline silicates. Although the alteration rates of amorphous silicate has not been experimentally measured, it seems possible that all the pre-existing interstellar amorphous silicates - if any - would have been transformed in clays during mild hydrothermal events.

To summarize this discussion on silicates in meteorites, the post-formation geological processes have likely erased the amorphous structures of interstellar precursors.

\subsection{Cometary silicates}

We discuss now the model results in the context of the amount of crystalline silicates present in the dust of longperiod comets such as Hale-Bopp. Dynamical considerations suggest that these comets formed mainly in the Uranus-Neptune zone. Our model shows that microscopic grains made of crystalline silicates present in the inner 
regions of the solar nebula are efficiently transported outwards to the comet formation zone by turbulence in timescales of a few $\sim 10^{4} \mathrm{yr}$.

The mass fraction of crystalline silicates in the dust of comet Hale-Bopp is estimated between $0.3-0.5$ to 0.9 (Table 1). Comparing with model results obtained in the outer regions (Table 4), we see that our model well explains the low range of measured values in the case of the warm nebula, regardless the crystallization law $t_{\text {ann }}$ adopted. On the other hand, the interpretation of the high degree of crystallinity $C_{\mathrm{c}}=90 \%$ inferred by Wooden et al. (1999) would require a still warmer nebula.

Radial mixing by turbulence is efficient as long as the grains are coupled to the gas. The decoupling occurs when particles agglomerate and grow up to sufficiently large sizes. There is no general consensus on the critical size for decoupling: it is about $1 \mathrm{~m}$ according to Weidenschilling (1997) and Supulver \& Lin (2000), while Stepinski \& Valageas (1996) advocate for centimetre, or even smaller, sizes. To be valid, our proposed scenario requires that timescales for grain growth from micrometre sizes to centimetre or metre sizes are not too short with respect to the diffusion timescale for radial mixing. Dust coagulation in protoplanetary disks is a complex problem, which is not yet well understood (see the review of Beckwith et al. 2000). The sticking process depends both on the chemical and physical properties of the grains, and on their relative velocity induced by thermal and turbulent motions, gas drag and sedimentation. Theoretical calculations predict that particle growth from sub-micron to metre sizes is very rapid and occurs in timescales of typically a thousand orbital revolutions, that is $\sim 10^{3}$ to $\sim 10^{4} \mathrm{yr}$ from 1 to 10 AU (Weidenschilling \& Cuzzi 1993; Stepinski \& Valageas 1996; see also Weidenschilling 1997, for results obtained at $30 \mathrm{AU})$. Such short coagulation timescales are in conflict with observational evidence that disks around $\mathrm{T}$ Tauri and Herbig Ae/Be stars of ages $\sim 10^{5}-10^{7}$ years are still dominated in mass by millimetre grains (see the review of Natta et al. 2000). On the other hand, fast coagulation seems a requisite to explain the presence of planets in the Solar System (Stepinski \& Valageas 1996). The coagulation timescales given above can be compared to our diffusion timescales. These latter are plotted as a function of radial distance and time in Fig. 5, for the warm nebula parameters which fit cometary silicate measurements. Coagulation timescales are somewhat lower, but by a factor of 5 at most, than the diffusion timescales computed at $t<10^{5}$ yr. Therefore, given actual uncertainties on grain growth, destruction processes by collisions (Benz 2000), and transport through turbulent eddies, we conclude that turbulent radial mixing of dust grains in the solar nebula is a plausible mechanism for explaining the large amount of crystalline silicates in comets.

In order to explain the $\mathrm{D} / \mathrm{H}$ ratios in cometary $\mathrm{H}_{2} \mathrm{O}$ and HCN, Hersant et al. (2001) concluded that microscopic grains of $\mathrm{H}_{2} \mathrm{O}$ and $\mathrm{HCN}$ ices, which subsequently accreted into cometesimals, condensed and were mixed together in the 10-20 AU range at times of typically $\sim 10^{5} \mathrm{yr}$.

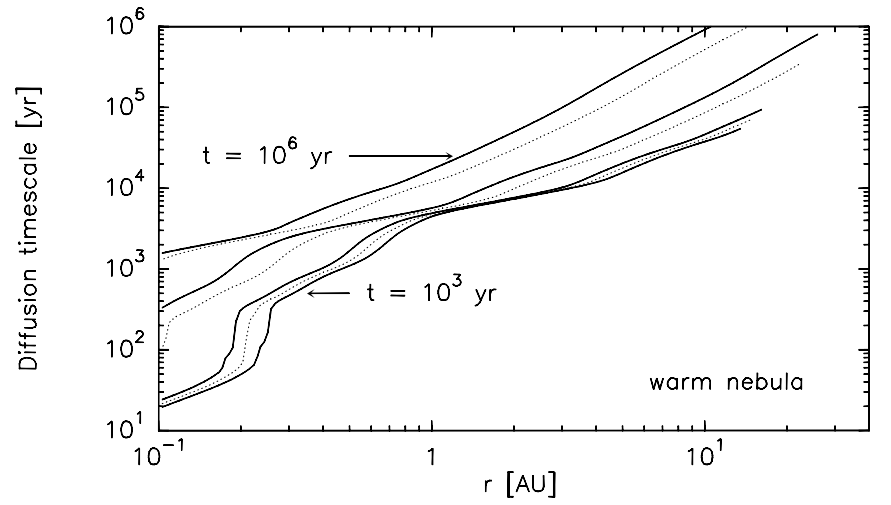

Fig. 5. Diffusion timescale $r^{2} / \nu_{\mathrm{t}}$ in the warm nebula as a function of time. Curves from bottom to top correspond to increasing times. Plain lines are for times $10^{3}, 10^{4}, 10^{5}$, and $10^{6}$ years. Dotted lines are for times $5 \times 10^{3}, 5 \times 10^{4}$, and $5 \times 10^{5}$ years.

Interestingly enough, this is also the time when the crystalline mass fraction in this heliocentric range has reached the plateau value (Fig. 3). In other words, at times of $\sim 10^{5} \mathrm{yr}$, silicates and icy particles with observed cometary properties were available in the comet formation zone. Their sticking and coagulation into kilometre-sized bodies could have then proceeded rapidly. With a vertical 1-D model run at $r=30$ AU which does not include turbulence, Weidenschilling (1997) shows that the formation of comets could have been completed in about $2 \times 10^{5} \mathrm{yr}$. To conclude, our nebula model permits us to consistently explain both siliceous and deuterium cometary composition by large scale turbulent radial mixing in the solar nebula. In turn, the amount of crystalline silicates in comets provides us with additional constraints on the parameters of the 2-D model of Hersant et al. (2001).

\subsection{Implications on the parameters of the 2-D solar nebula model}

One of our primary goals was to investigate whether solar nebulae models which fit deuterium enrichments in primitive Solar System bodies (Hersant et al. 2001), would be able to explain the amount of crystalline silicates measured in cometary dust. In fact, the present analysis based on three selected nebulae shows that producing the amount of cometary crystalline silicates requires the warm nebula. Since a number of other warm nebulae fitting deuterium enrichments can be generated by the 2-D model, the relative proportion of crystalline silicates in comets can be used as an additional constraint to the selection of nebulae. Based on the observational data (Table 1), we adopted the value of $C_{\mathrm{c}}=0.3$ for this constraint. A number of nebulae were then calculated, as in Hersant et al., each one defined by the initial accretion rate $\dot{M}_{0}$ and radius $R_{\mathrm{D} 0}$ of the nebula, and the coefficient of turbulent viscosity $\alpha$. The equations of diffusion 6 and 7 were then integrated as a function of radial distance and time, using the surface density and temperature radial profiles of these nebulae. Nebulae which 
do not provide adopted constraints were rejected. The ranges of acceptable nebula parameters fitting both $\mathrm{D} / \mathrm{H}$ and $C_{\mathrm{c}}$ measurements are substantially reduced with respect to those obtained by Hersant et al. (2001). They are: $0.008<\alpha<0.01,7 \times 10^{-6}<\dot{M}_{0}<10^{-5} M_{\odot} \mathrm{yr}^{-1}$, and $12.4<R_{\mathrm{D} 0}<14.8$ AU.

The derived ranges of acceptable $\alpha, \dot{M}_{0}$ and $R_{\mathrm{D} 0}$ values are very small and should not be taken too seriously. First, the adopted physical and chemical constraints might not be fully appropriate. Indeed, there are still large uncertainties on $\mathrm{D} / \mathrm{H}$ values and silicate composition in comets. More important, among physical constraints adopted by Hersant et al. (2001), the initial mass of the solar nebula was limited to masses smaller than $0.3 M_{\odot}$, in order to satisfy the criterion of gravitational stability of the disk, and the radius of the solar nebula was forced to reach Neptune distance in the first $2.5 \times 10^{5} \mathrm{yr}$. These assumptions, which could be certainly somewhat relaxed, result in the rejection of nebulae with warm temperatures in their early life and constrain the initial radius of the nebula to a small range. The second point to consider is that the results are model dependent and obtained under several assumptions. Among these, the value of the Prandtl number, which is not well known, has a strong influence on the derived $\alpha, \dot{M}_{0}$ and $R_{\mathrm{D} 0}$ ranges (Hersant et al. 2001). The uncertainties on the timescales for isotopic exchanges and thermal annealing are significant. Finally, the validity of the disk model may be also questioned. Standard $\alpha$-disks are known to be not extended and warm enough to explain observations of circumstellar disks. Many alternative and controversial solutions have been proposed and are beyond the scope of the present paper. The initial high accretion rate of $\sim 10^{-5} M_{\odot} \mathrm{yr}^{-1}$ that we derived would correspond to that of Class 0 protostars when mass infall from the envelope is feeding the disk (André et al. 2000). Admittedly, this stage of the early nebula is not well treated in our model and might affect the initial and boundary conditions of our transport equations. However, we must point out that time-dependent models describing the early stages of the solar nebula when most of its chemical evolution occurs, are not yet available.

\section{Summary}

This paper presents a model to explain the presence of a high amount of crystalline silicates in comets. The proposed scenario is that crystalline silicates formed in the inner warm regions of the solar nebula by the thermal annealing of amorphous silicates. They were then transported out to the comet formation zone by turbulent diffusion. This paper follows those of Drouart et al. (1999), Mousis et al. (2000) and Hersant et al. (2001), which interpret the variety of deuterium enrichments observed in primitive objects of the Solar System by isotopic exchange in the solar nebula and turbulent radial mixing.

Our study makes use of the 2-D evolutionary solar nebula model developed by Hersant et al. (2001). It takes into account the rapid evolution of temperatures and pressures during disk evolution. Such an approach is necessary to investigate quantitatively whether the proposed scenario is plausible, since both thermal annealing and turbulent mixing processes are strongly temperature dependent.

Using solar nebula parameters which fit $\mathrm{D} / \mathrm{H}$ ratios in comets, we show that turbulent diffusion is an efficient mechanism for mixing amorphous and crystalline grains in the comet formation zone. The timescale required for radial mixing is typically $\sim 1-5 \times 10^{4} \mathrm{yr}$, depending on the solar nebula model. These values are comparable, within a factor of a few, to theoretical expectations concerning the characteristic timescales for particle growth in the solar nebula. It is also well below the time needed to form kilometer-sized comets from a population of microscopic grains (Weidenschilling 1997). Therefore, given actual uncertainties on grain growth in the solar nebula, it seems likely that fast dust coagulation did not preclude largescale radial mixing, allowing the transport of crystalline silicates from inner to outer regions.

One of the aims of our modelling was to investigate whether the solar nebulae selected by Hersant et al. (2001) to fit cometary $\mathrm{D} / \mathrm{H}$ ratios would also explain quantitatively the high amount of crystalline silicates. Our calculations show that fitting both observational constraints is possible, provided that we use model parameters which generate warm nebulae. Using the mass fraction of crystalline silicates estimated in comet Hale-Bopp as an additional constraint to the model of Hersant et al. (2001), we derived the ranges of nebula parameters $\alpha, \dot{M}_{0}$ and $R_{\mathrm{D} 0}$ which fit both cometary and silicate composition. The ranges of acceptable parameters are small and may not represent the number of uncertainties and assumptions in our model. However, this study qualitatively demonstrates the need of high temperatures in an extended zone of the solar nebula, to explain both $\mathrm{D} / \mathrm{H}$ and the properties of cometary silicates. In addition, it shows that, in essence, the amount of crystalline silicates in comets should permit us to check the validity of any model of the solar nebula in which the temperature distribution is calculated. More conclusive studies will have to await for the availability of more realistic time-dependent models for solar nebula early evolution.

Our model predicts that, in a few $10^{4} \mathrm{yr}$, the crystalline/amorphous silicates ratio reaches the same value in almost the whole nebula, except in the innermost regions. This might imply that all comets formed in the turbulent part of the solar nebula could have the same crystalline/amorphous silicates ratio, a result not in conflict with available data on long-period comets (Sect. 2). However, firm conclusions should await for more detailed models which include grain coagulation, since this process affects the efficiency of radial mixing by turbulence. In addition, measurements of the crystalline/amorphous silicates ratio in comets are still too sparse to allow detailed comparison with model predictions. Data on Jupiter family comets coming from the Kuiper Belt are specifically required. They may reveal a lower content of crystalline 
silicates, if they were formed in a non-turbulent part of the outer nebula.

Acknowledgements. We thank D. Fabian, for providing us with laboratory results before publication, and P. Michel and J. R. Brucato for enlightening discussions. This work was supported by the Programme national de planétologie de l'Institut national des sciences de l'univers (INSU) and the Centre de la recherche scientifique (CNRS).

\section{References}

André, P., Ward-Thompson, D., \& Barsony, M. 2000, in Protostars and Planets IV, ed. V. Mannings, A. P. Boss, \& S. S. Russel (The University of Arizona Press, Tucson), 59

Ashworth, J. R., Mallinson, L. G., Hutchinson, R., \& Biggar, G. M. 1984, Nature, 308, 259

Beckwith, S. V. W., Henning, T., \& Nakagawa, Y. 2000, in Protostars and Planets IV, ed. V. Mannings, A. P. Boss, \& S. S. Russel (The University of Arizona Press, Tucson), 553

Benz, W. 2000. Space Sci. Rev., 92, 279

Bouwman, J., Meeus, G., de Koter, A., et al. 2001, A\&A, 375, 950

Bradley, J. P., Brownlee, D. E., \& Veblen, D. R. 1983, Nature, 301, 473

Bradley, J. P., Humecki, H. J., \& Germani, M. S. 1992, ApJ, 394,643

Bradley, J. P., Keller, L. P., Snow, T., et al. 1999, Science, 285, 1716

Brucato, J. R., Colangeli, L., Mennela, V., Palumbo, P., \& Bussoletti, E. 1999a, P\&SS, 47, 773

Brucato, J. R., Colangeli, L., Mennela, V., Palumbo, P., \& Bussoletti, E. 1999b, A\&A, 348, 1012

Cassen, P. 1996, Meteoritics \& Planetary Science, 31, 793

Cassen, P. 2001, Meteoritics \& Planetary Science, 36, 671

Chick, K. M., \& Cassen, P. 1997, ApJ, 477, 398

Colangeli, L., Brucato, J. R., Ferrini, et al. 1999, Adv. Space Res., 23, 1243

Colangeli, L., Mennela, V., Rotundi, A., Palumbo, P., \& Bussoletti, E. 1995, A\&A, 293, 927

Colangeli, L., Mennela, V., Rotundi, A., Palumbo, P., \& Bussoletti, E. 1996, A\&A, 312, 643

Crovisier, J., Brooke, T. Y., Leech, K., et al. 2000, in Thermal emission spectroscopy and analysis of dust disks, and regoliths, ed. M. L. Sitko, A. L. Sprague, \& D. K. Lynch, Astron. Soc. Pac. Conf. Ser., 196, 109

Crovisier, J., Encrenaz, T., Lellouch, E., et al. 1999, in The Universe seen by ISO, ed. P. Cox, \& M. F. Kessler, ESA SP427,161

Crovisier, J., Leech, K., Bockelée-Morvan, D., et al. 1997, Science, 275, 1904

Demyk, K., Jones, A. P., Dartois, E., Cox, P., \& d'Hendecourt, L. 1999, A\&A, 349, 267

Drouart, A., Dubrulle, B., Gautier, D., \& Robert, F. 1999, Icarus, 140, 129

Dubrulle, B., Morfill, G., \& Sterzik, M. 1995, Icarus, 114, 237

Duschl, W. J., Gail, H.-P., \& Tscharnuter, W. M. 1996, A\&A, 312,624

Fabian, D., Jäger, C., Henning, Th., Dorschner, J., \& Mutschke, H. 2000, A\&A, 364, 282

Gail., H.-P. 1998, A\&A, 332, 1099
Gail., H.-P. 2001, Radial mixing in protoplanetary accretion disks. I. Stationary disc models with annealing and carbon combustion, A\&A, 378, 192

Gail., H.-P., \& Sedlmayr, E. 1999, A\&A, 347, 594

Hallenbeck, S. L., Nuth, J. A., \& Daukantas, P. L. 1998, Icarus, 131, 198

Hanner, M. S. 1999, Space Sci. Rev., 90, 99

Hanner, M. S., Brooke, T. Y., \& Tokunaga, A. T. 1998, ApJ, 502,871

Hanner, M. S., Gehrz, R. D., Harker, D. E., et al. 1997, Earth, Moon, and Planets, 79, 247

Hanner, M. S., Lynch, D. K., \& Russel, R. W. 1994, ApJ, 425, 274

Hartmann, L., Calvet, N., Gullbring, E., \& D'Alessio, P. 1998, ApJ, 495, 385

Hawley, J. F., Smarr, L. L., \& Wilson, J. R. 1984, ApJ, 55, 211

Hayward, T. L., Hanner, M. S., \& Sekanina, Z. 2000, ApJ, 538, 428

Hersant, F., Gautier, D., \& Huré, J.-M. 2001, ApJ, 554, 391

Hewins, R. H. 1997, Ann. Rev. Earth. Planet. Sci., 25, 61

Hewins R. H., \& Radomsky, P. M. 1990, Meteoritics, 25, 309

Huré, J.-M. 2000, A\&A, 358, 378

Huré, J.-M., \& Galliano, F. 2001, A\&A, 366, 359

Knacke, R. F., Fajardo-Acosta, S. B., Tedesco, C. M., et al. 1993, ApJ, 418, 440

Lenzuni, P., Gail, H.-P., \& Henning, Th. 1995, ApJ, 447, 848

Lofgren, G. E., \& Russell, W. J. 1988, Ceochim. Cosmochim. Acta, 50, 1715

Makalkin, A. B., \& Dorofeyeva, V. A. 1991, Izv. Earth Phys., 27,650

Malfait, K., Waelkens, C., Waters, L. B. F. M., et al. 1998, A\&A, 332, L25

Molster, F. J., Yamamura, I., Waters, L. B. F. M., et al. 1999, Nature, 401, 563

Morfill, G. E., \& Völk, H. J. 1984, ApJ, 287, 371

Mousis, O., Gautier, D., Bockelée-Morvan, D., Robert, F., \& Dubrulle, B. 2000, Icarus, 148, 513

Natta, A., Grinin, V. P., \& Mannings, V. 2000, in Protostars and Planets IV, ed. V. Mannings, A. P. Boss, \& S. S. Russel (The University of Arizona Press, Tucson), 559

Neufeld, D. A., \& Hollenbach, D. J. 1994, ApJ, 428, 170

Nuth, J. A., \& Donn, B. 1982, ApJ, 257, L103

Radomsky, P. M., \& Hewins, R. H. 1990, Geochim. Cosmochim. Acta, 54, 3475

Ruden, S. P., \& Lin, D. N. C. 1986, ApJ, 308, 883

Ruden, S. P., \& Pollack, J. B. 1991, ApJ, 375, 740

Scott, E. R. D. 1979, Mineral. Mag., 43, 415

Shakura, N. L., \& Sunyaev, R. A. 1973, A\&A, 24, 337

Shu, F. H., Tremaine, S., Adams, F. C., \& Ruden, S. P. 1990, ApJ, 358

Stepinski, T. F., \& Valageas, P. 1996, A\&A, 309, 301

Supulver, K. D., \& Lin, D. N. C. 2000, Icarus, 146, 525

Wilson, J. R. 1978, in Source of Gravitational Radiation, ed. L. Smarr (Cambridge University Press, Cambridge), 423

Tsuchiyama, A., \& Nagahara, H. 1981, in Proceedings of the 6th symposium on Antartic Meteorites, ed. T. Nagata, Mem. Nat. Inst. Polar Res., Spec. Issue, 20, 175

Weidenschilling, S. J. 1997, Icarus, 127, 290

Weidenschilling, S. J., \& Cuzzi, J. N. 1993, in Protostars and Planets III, ed. E. H. Levy, \& J. I. Lunine (The University of Arizona Press, Tucson), 1031

Wooden, D. H., Harker, D. E., Woodward, C. E., et al. 1999, ApJ, 517, 1034 